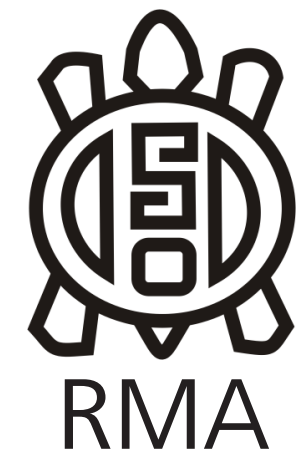

Dossier

\title{
Piedras de rayo: análisis de rocas desde una mirada integral
}

\author{
Lightning stones: lithics analysis from an holistic perspective
}

\author{
Jorge E. Cabral Ortíz*, Cecilia Mercuri**, Pilar García De Cecco*** y \\ Federico G. Mendoza***
}

* Museo Arqueológico de Cachi. E-mail: jorgestebancabral@gmail.com

** ICSOH/ CONICET. E-mail: ce_mercuri@yahoo.com.ar

***Universidad Nacional de Salta. E-mail: piilu.5@hotmail.com; fedegmendoza@gmail.com

\begin{abstract}
Resumen
Desde hace un tiempo, se observa cada vez mayor interés por la combinación de distintos tipos de análisis para una mejor comprensión de los usos, aprovechamiento y producción de instrumentos y materias primas líticas. Así, se potencian diferentes líneas de evidencia desde una perspectiva integral de abordaje del instrumental, propiciando investigaciones que planteen las ventajas de la complementariedad y los análisis holísticos. En Diciembre de 2017 se llevaron a cabo excavaciones de rescate arqueológico en el sitio Cac8 conocido como Capilla de la Aguada, en Cachi, Salta. Estas actividades se encuentran enmarcadas en un proceso de diálogo con las organizaciones comunales a través del cual surgen encuentros que ponen en relieve una construcción conjunta del conocimiento, donde el patrimonio material adquiere un sentido para la comunidad. En este contexto, el estudio del material lítico se realizó mediante un abordaje holístico e integral en el que se contemplan las metodologías tradicionales de análisis tecno- tipológico combinadas con el registro de entrevistas a los pobladores. De acuerdo al estudio preliminar, se propone un evento de carácter ritual en el que se destacan la presencia de 10 piedras pulidas de cuarzo, siendo prácticamente los únicos artefactos líticos recuperados en excavación, en la cual el material cerámico es muy abundante.
\end{abstract}

Palabras clave: Piedras de rayo; Análisis lítico; Etnografía; Patrimonio arqueológico; Cachi.

\begin{abstract}
For some time, there has been an increasing interest in the combination of different types of analysis for a better understanding of the uses, exploitation and production of lithic raw materials and instruments. Thus, different lines of evidence are promoted from an integral perspective of approaching the instruments, fostering research that raises the advantages of complementarity and holistic analysis. On December 2017, archaeological rescue excavations were carried out at the Cac8 site known as Capilla de la Aguada, in Cachi, Salta. These activities are framed in a process of dialogue with the community organizations through which encounters arise that highlight a joint construction of knowledge, where material heritage acquires a meaning for the community. In this context, the study of the lithic material was carried out through a holistic and integral approach in which the traditional methodologies of techno-typological analysis were combined with the register of interviews with the settlers are contemplated. According to the preliminary study, it is proposed an event of a ritual nature in which the presence of 10 polished quartz stones stands out, being practically the only lithic artifacts recovered in excavation, in which the ceramic material is very abundant.
\end{abstract}

Keywords: Lighting stones; Lithic analysis; Ethnography; Archaeological heritage; Cachi.

En un contexto global en el cual el patrimonio y su conservación están cobrando relevancia, se van potenciando los análisis de materiales desde perspectivas integrales, lo cual propicia las investigaciones que plantean las ventajas de la complementariedad y los análisis holísticos.

La narración del pasado en el NOA se fue construyendo en una diversa serie de relatos los cuales muchas veces se utilizaron (y se utilizan) como herramienta de legitimación, de sustento ideológico para crear, sostener y/o revertir situaciones o coyunturas sociopolíticas y económicas específicas (Manasse 2012). El conocimiento arqueológico es social y, como todo discurso sobre el pasado, interviene y se inscribe en una determinada realidad social presente (cf. Manasse 2012, Hodder 1986; Mc Guire 1987; Shanks y Tilley 1987; entre otros). En el valle Calchaquí Norte, las comunidades articulan su vida cotidiana con 
los objetos arqueológicos, identificándolos desde la práctica y la experiencia. Por ejemplo, los miembros de la comunidad dan cuenta de la existencia de los sitios arqueológicos, refiriéndose a ellos con el término de "Antigal", reconociéndolos no solo como los lugares en el que habitaban los antiguos, sino también en relación al conjunto de tiestos y pircas que se observan desde la superficie (Cabral y Rivolta 2017).

Ya desde fines del siglo XX, en nuestro país se comienzan a discutir conceptos arraigados en la práctica arqueológica. Así, se proponen nuevas reflexiones críticas sobre el involucramiento de la arqueología en la sociedad y los efectos políticos de sus producciones (Delfino et al 2016). Lo que se pretende es superar el rol de "documentador del pasado" para atender a la contingencia del trabajo arqueológico y a la multivocalidad de las interpretaciones del pasado (Salerno 2013). En este marco, cobran relevancia las tareas de divulgación, extensión, educación, transferencia e intervenciones de rescate arqueológico, debido a que se busca escuchar y hacer partícipes las demandas que surgen de distintas situaciones que afectan los bienes arqueológicos (Delfino et al 2016). A mediados de la década de 1980, Delfino y Manasse (1986) plantean la necesidad de articulación entre el arqueólogo y las sociedades que se ven involucradas de algún modo en su trabajo. Esta línea argumentativa se concreta con la definición de una Arqueología Socialmente Útil (Delfino y Rodríguez 1989), en la cual el vínculo entre arqueología y sociedad conforma la base, estableciéndose un compromiso social y político (Manasse 2012). Se constituye así una práctica donde se articulan saberes (nativos/campesinos y científicos) pudiéndose diseñar estrategias transformadoras de realidades sociales, culturales y políticas que lleven a un empoderamiento que aporte a la reafirmación de derechos de las comunidades (cf. Barale y Delfino 2007, Manasse 2012, Delfino et al. 2013, Delfino et al 2016, entre otros). Entonces, los resultados de los estudios arqueológicos pueden constituir un aporte concreto y directo a requerimientos sociales y culturales actuales de las comunidades locales, vinculadas a procesos de construcción histórica e identitaria y a la problemática territorial y de gestión del patrimonio arqueológico (Manasse 2012).

A partir de estas miradas consideramos útil una metodología que recurra a diversos tipos de fuentes y una perspectiva basada en la construcción colectiva de conocimientos que, desde una postura dialógica, dé cuenta de una arqueología que parta de la articulación de los distintos saberes en tensión, en un lugar donde las memorias del pasado se vuelven una parte activa en el proceso de constitución de sujetos históricos presentes (Jofré et al. 2008). Esto se debe a que la materialidad arqueológica es parte del espacio y también del campo de acción donde se desarrolla la trama social y cultural actual.
En diciembre de 2017 se realizó una excavación de rescate arqueológico en el sitio conocido como Capilla de la Aguada (SsalCac8), ubicado en la margen derecha de Río Las Cuevas en la localidad de Cachi, Salta. El mismo fue relevado en la década de 1970 por Pío Pablo Díaz, quien lo describió como un conglomerado de estructuras entre las que se destacan montículos con tumbas circulares y algunos muros de roca canteada, donde se halló un entierro en una vasija con un "ajuar" que incluía cerámica de tipo inca-paya (Diaz, 1972). Pese a lo descrito por Díaz, el sitio se encuentra en un estado de deterioro tal, que no es posible observar el patrón monticular ni las estructuras mencionadas por el mismo. Esta situación está vinculada tanto a factores ambientales como sociales y determina que los recintos se encuentren sepultados imposibilitando la capacidad de observarlos en superficie. En relación a ello, las actividades aquí descritas se realizaron a raíz de que un comunero, al comenzar tareas relativas a la construcción de un pozo sanitario, realizó el hallazgo de una estructura de piedra de unos 8 metros de diámetro. La intervención pudo ser llevada a cabo gracias a las actividades que se vienen desarrollando en el marco de un proceso de diálogo con las organizaciones comunales, tales como encuentros que ponen en relieve una construcción conjunta del conocimiento, donde el patrimonio material adquiere un sentido para la comunidad.

Se excavaron 2 cuadrículas (A y B) de $1.80 \times 1.60 \mathrm{~m}$. en las márgenes noroeste y suroeste del pozo que se abrió para comenzar con el trabajo de construcción previamente mencionado. La excavación se realizó siguiendo los niveles naturales y culturales y con el objetivo de registrar el contexto asociado a los objetos que quedaron al descubierto por acción de la maquinaria en el sitio. A los $69 \mathrm{~cm}$ de profundidad, se detectó un piso de ocupación asociado a un recinto habitacional caracterizado, en la cuadrícula B, por la presencia de una lente de cenizas, 10 piedras pulidas de forma ovoide y dos artefactos líticos alargados, dispuestos junto al cuerpo de una vasija tosca peinada sin base, una escudilla con asa en cinta adherida y una pequeña vasija con pico vertedor, ambas de tipo Yavi (Figura 1), asignables a los siglos XV- XVII (entre momentos de conquista inca e hispana). La interpretación de contextos similares (cf. Lynch et al 2015) ha seguido un camino meramente funcional, donde las piedras ovoides pulidas serían pulidores de cerámica.

Por el momento carecemos de una interpretación más completa del caso de estudio por encontrarse los materiales bajo análisis.

\section{Análisis integral}

Para el estudio del material lítico se optó por un abordaje holístico e integral en el que se contemplaron las metodologías tradicionales de análisis tecno- tipológico 


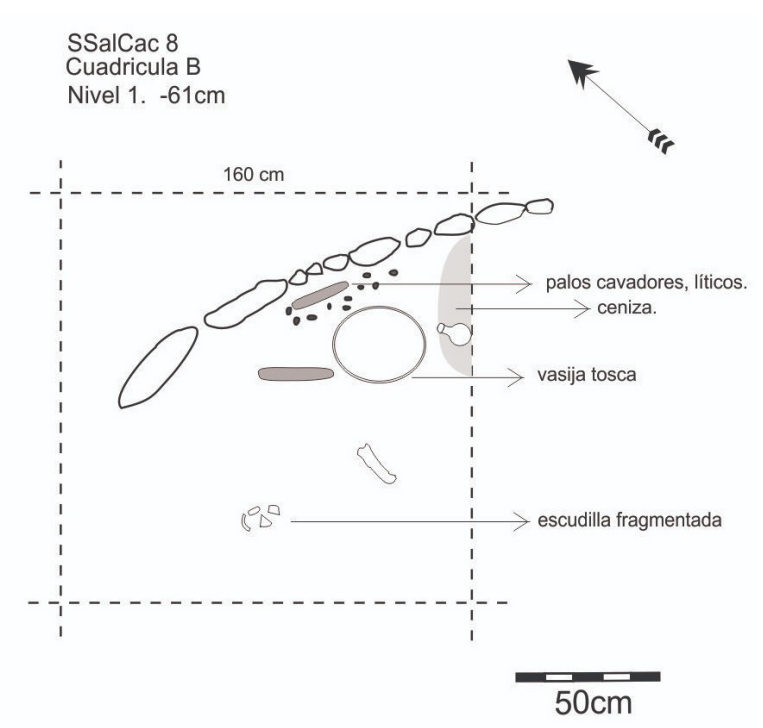

Figura 1. Planta de la excavación de la cuadrícula B. En el esquema se señalan las piezas tratadas en este artículo.

Figure 1. Grid excavation plant B. In the scheme are shown the objects trated in this article.

(Aschero 1975 y 1983) combinadas con el registro de entrevistas a los pobladores. La elección de dicho enfoque estuvo determinada por su potencial para la elaboración de interpretaciones y herramientas para la construcción de saberes que no derivasen en la alienación de la comunidad con su pasado (Manasse et al 2019). En este sentido y, en el marco del proceso de gestión patrimonial emprendido por la comunidad de La Aguada, nos propusimos implementar un abordaje en el que el saber técnico-científico se articulara con el saber local logrando nuevas formas de comprender las materialidades (Cabral Ortíz y Rivolta 2017). Para ello se apuntó a una construcción conjunta con los actores involucrados y, en particular, con los que allí habitan, lo cual implicó un intercambio de saberes que contribuyó tanto a la interpretación en arqueología como a los/as comuneros/as en un trabajo de objetivos compartidos (Cabral Ortíz y Rivolta 2017, Manasse et al 2019). Así, el trabajo tomó el carácter de una etnografía arqueológica (Menezes 2015), buscando entender las cosmologías y representaciones de las comunidades sobre los objetos arqueológicos y sus narrativas sobre el pasado, promoviendo un espacio intercultural de diálogo permanente entre el equipo arqueológico y la comunidad (Menezes 2015). Asimismo, en estas tareas se está intentando integrar a las comunidades en todas las etapas de investigación arqueológica, tratando de dotar a las comunidades con instrumentos útiles para el manejo de su patrimonio arqueológico (Cabral Ortíz y Rivolta 2017).

\section{Análisis tecnomorfológico}

Se analizaron 15 artefactos procedentes de la cuadrícula $B$, del rescate efectuado en Capilla de la Aguada, realizado en diciembre de 2017 a pedido de la comunidad de La Aguada. En el protocolo de análisis se siguió mayormente la propuesta de Aschero 1975 y 1983. Sin embargo, dadas las características del conjunto analizado, se tuvieron en cuenta variables que tenían que ver principalmente con la materia prima y los rastros complementarios (cf. Mercuri et al 2012) (Tabla 1.).

Los artefactos recuperados se tratan principalmente de artefactos pulidos (90\%). La mecánica por la cual se logró este efecto es un punto de discusión interesante, ya que durante el trabajo con la comunidad surgieron algunos comentarios que permiten generar hipótesis al respecto.

Se registraron 10 artefactos de cuarzo en diversos tonos, desde el blanco al amarillo y el rosa, aunque en un $80 \%$ son blancos (Figura 2). La coloración es el atributo que presenta mayor variabilidad en el conjunto. Todas estas rocas son locales. La forma general del contorno es redondo u oval (Figura 3).

En algunos casos (30\%) presentan melladuras, marcas de piqueteado y/o golpes (sensu Aschero 1975) en ambos o uno de los extremos. Por el momento no pudimos determinar si los mismos se deben al uso o a la formatización de estos artefactos. En relación a este punto, en una de las piezas se observan negativos de lascado, evidenciando que la pieza no ha concluido de formatizarse por pulido (Figura 4). Es la única pieza amorfa (sensu Aschero 1975) de la colección.

En relación al tamaño, sus medidas son tendientes a uniformes, siendo el largo promedio de unos $3,3 \mathrm{~cm}, 2,8$ de ancho y 2,2 cm de espesor (Figuras 5 y 6 ). En ambos gráficos observamos cómo la muestra se concentra en nubes, demostrándose la regularidad en cuanto a tamaño.

Otros dos artefactos de morfología similar a los anteriores

\begin{tabular}{|c|c|c|c|c|c|c|c|c|c|}
\hline $\begin{array}{c}\text { materia } \\
\text { prima }\end{array}$ & color & $\begin{array}{c}\text { forma } \\
\text { general } \\
\text { del } \\
\text { contorno }\end{array}$ & largo & ancho & espesor & formatización & $\begin{array}{c}\text { rastros } \\
\text { complementarios }\end{array}$ & observaciones & foto \\
\hline
\end{tabular}

Tabla 1. Ficha utilizada en el análisis tecno-morfológico de las piezas.

Table 1. Variables used in the techno-morphological analysis. 


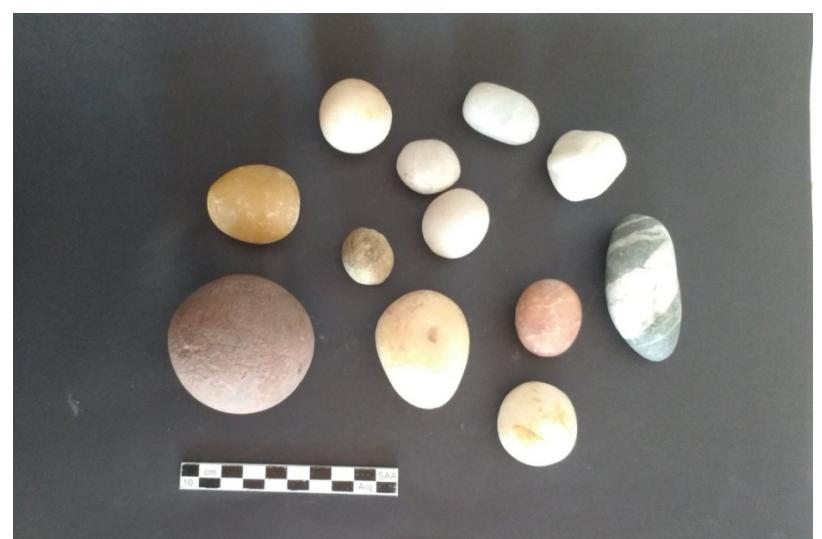

Figura 2. Variabilidad en cuarzos locales.

Figure 2. Variability in local quartz.

y recuperados en el mismo contexto son un pequeño artefacto en una roca sedimentaria de color ocre amarillento, en forma esférica. A diferencia de las otras rocas de la colección, esta es opaca, pero las medidas y la forma son las mismas, aunque presenta alguna irregularidad en su pulido. La otra pieza es de cuarcita rojo oscuro, esférica aunque presenta una cara más plana. Tiene $6 \mathrm{~cm}$ de diámetro. Se registraron melladuras y marcas de piqueteo generando irregularidades en la superficie plana. Un poco más lejos de esta concentración, se recuperaron dos artefactos líticos alargados o 'palos cavadores' (ver pe. Pérez 2008). Se trata de artefactos en andesita gris oscuro, en forma de cilindro de unos $5 \mathrm{~cm}$ de diámetro (ambos) y 30 y $25 \mathrm{~cm}$ de largo. Este último presenta un rebaje a los $10 \mathrm{~cm}$ de un extremo.

\section{Aproximación etnográfica}

Desde el año 2013, nuestra tarea de investigación viene transitando diferentes experiencias surgidas del dialogo con los miembros de las comunidades Daguita Calchaquí del paraje La Aguada. En este marco, tanto arqueólogos como comuneros, consensuamos las tareas de excavación arqueológica con el objetivo de recuperar los objetos para luego ser exhibidos en el centro de interpretación "Antigal El Tero". Este espacio, consiste en un museo comunitario gestionado por las organizaciones de pueblos originarios.

Como parte de este proceso en el que la labor arqueológica

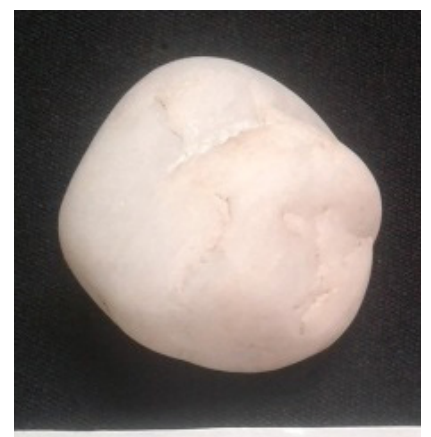

Figura 4. Pieza amorfa en cuarzo, se observan posibles negativos de lascado en la parte superior.

Figure 4. Amorphous piece in quartz, posible lacquered negative are observed in the upper part.

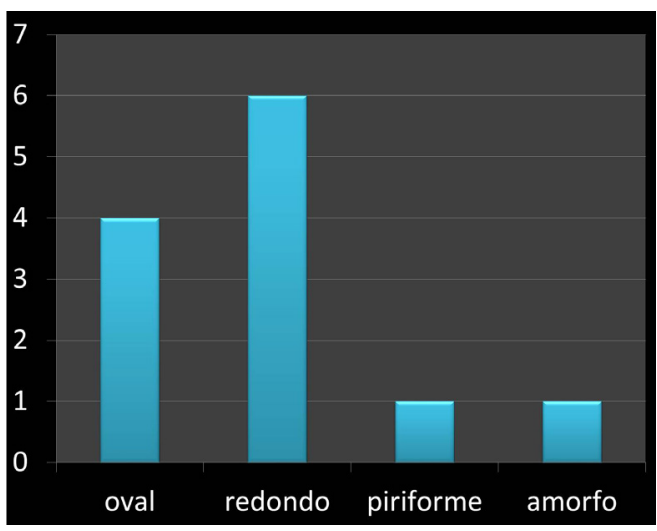

Figura 3. Forma general del contorno.

Figure 3. General contour shape.

es interpelada, optamos por una mirada antropológica con el objetivo de comprender los múltiples significados en torno a los objetos arqueológicos rescatados. A partir de las excavaciones en conjunto con los comuneros que surgieron las primeras interpretaciones respecto a los objetos líticos provenientes de la cuadricula A. Fue así que, a través de los discursos que emergieron en las instancias de excavación de manera informal, pudimos observar un sentido con el que eran identificados algunos objetos arqueológicos. Por ello optamos por dos estrategias, por un lado, realizar un conjunto de entrevistas semi-estructuradas dirigidas a 5 mujeres mayores (de entre 50 a 60 años) y, por otro, conformar un espacio de diálogo a partir de una instancia de conversatorio. Con ello, buscamos discutir los contextos arqueológicos excavados, propiciando la participación de los actores que interpelan la mirada científica desde el saber local. Consideramos a estas técnicas como las propicias para generar un espacio dialógico, a partir de la cual podemos detectar las situaciones en que se expresan y generan los universos culturales y sociales en su compleja articulación y variedad (Guber, 2011).

En el Valle Calchaqui Norte, los objetos arqueológicos son interpretados desde la experiencia y reconocidos según su utilización en la actualidad. Por ejemplo, las vasijas toscas son descriptas como tinajas que sirvieron para la cocción de ciertas bebidas o para el almacenamiento de agua o granos (Cabral, Rivolta 2017). Siguiendo ésta misma lógica, los comuneros identificaron a uno de los objetos líticos pulidos de forma ovoide como una piedra del rayo. Esta piedra de color rojiza es utilizada como parte de una práctica curativa y "sirve para sacar el susto y sanar el corazón". Asimismo, las rocas de cuarzo fueron identificadas como las que usan los ancianos para raspar la piedra rojiza junto a un poco de agua que debe tomarse por las mañanas en el proceso de curación: "es mejor si la deja con agua de rocío y después la raspa con la blanquita y la toma a la mañana en ayunas...". Estos sentidos, sobre esos objetos encuentran similitud con las miradas de las 


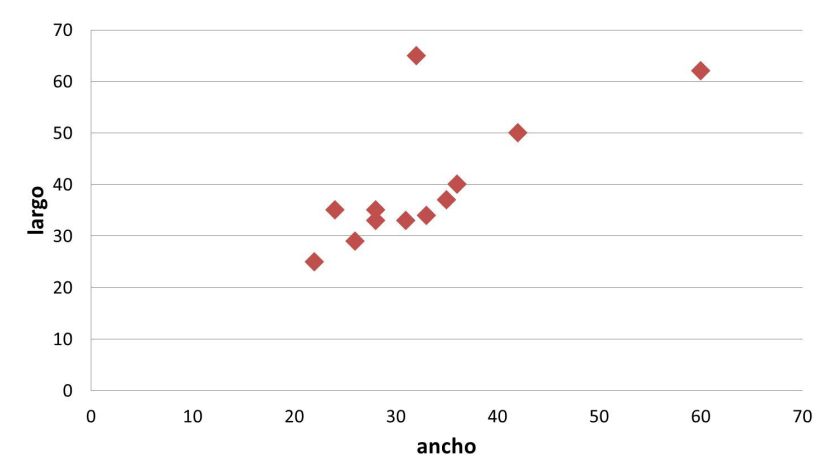

Figura 5. Medidas. Relación largo ancho.

Figure 5. Lenght-width ratio.

comunidades aimaras del altiplano boliviano, quienes usan estas piedras para curar el susto provocado por el rayo o el mal de corazón (Juárez, 1997).

\section{Discusión}

En este diálogo surgieron diferentes aspectos centrados en los objetos arqueológicos. Diferencias que parten de una manera distinta de concebir, explicar y constituir un saber local que interpela la mirada científica. Narrativas que revelan modos de construir diferentes sentidos determinados por el lugar de enunciación, un lugar no solo político y/o cultural sino también epistémico (Walsh 2007, en Cabral Ortíz y Rivolta 2017).

A partir de los conocimientos adquiridos y en base al registro etnográfico, las piedras ovoides recuperadas en el sitio Cac8 podrían ser interpretadas como piedras del rayo las cuales según los comuneros son utilizadas para curar enfermedades del espíritu (aunque no se admite abiertamente el uso) (ver también Arguedas 1951, Bugallo 2009, Vilca 2009). Sin embargo, esta aproximación se contrapone con la mirada arqueológica, a partir de la cual estos objetos pueden ser considerados como pulidores.

Es así que, partiendo de los análisis que propician la complementariedad de conocimientos de diversas líneas de investigación, proponemos que estos objetos pueden ser comprendidos en el marco de prácticas rituales dadas las características de su asociación contextual y la información incorporada a partir de la mirada local en vistas de profundizar la interpretación arqueológica. Los conocimientos locales son construidos por la interacción cotidiana de los grupos con su entorno, por lo que su inclusión al proceso de investigación científica reviste de un gran potencial para nuestras interpretaciones. En este sentido, destacamos que la complementariedad de conocimientos enriquece a los estudios arqueológicos, pero también puede servir para reforzar los procesos de identidad comunitaria, ya que los patrimonios se construyen socialmente a través de la legitimación de ciertos referentes materiales y simbólicos (Aljanati et

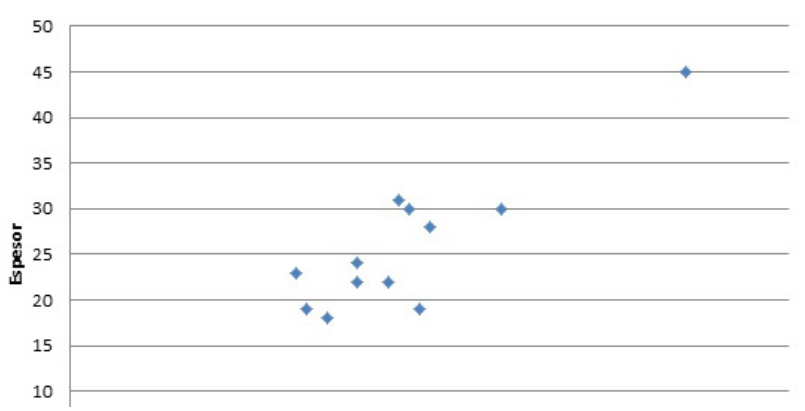

Figura 6. Medidas. Relación espesor ancho.

Figure 6. Width-thickness ratio.

al 2017). El subsumir la arqueología exclusivamente al objeto desenfoca el sentido social de la misma.

El diálogo con la comunidad, también nos permitió aproximarnos a la confección de los artefactos. La mecánica de la formatización, fue un punto de discusión interesante, ya que la materia prima elegida (cuarzo) es muy dura (7 en la escala de Moss) como para ser pulida por la acción del frotado constante en las manos, no obstante, si se frotan dos o más rocas a la vez, tal vez se consiga este resultado. Indagar en este aspecto es una hipótesis de trabajo a futuro.

En la localidad de Cachi, fue interesante percibir que la experiencia con la comunidad de La Aguada estaría poniendo en cuestión la penetración de los discursos oficiales, ya que en el diálogo surgido a partir de la excavación y las piezas arqueológicas se manifiesta que las personas continúan significando los objetos 'arqueológicos' a partir de su experiencia, desconociendo el relato científico construido desde las instituciones (piedras de rayo vs. pulidores). En este contexto, se puede afirmar un lugar de resistencia atravesado por diversas disputas (ej. acceso a la tierra), a partir de las cuales se logran constituir/ construir otros espacios de expresión en el que los objetos arqueológicos (patrimonio) se constituyen/construyen como nuevas materialidades al significar la preexistencia en el territorio por el que se lucha (Cabral Ortíz y Rivolta 2017).

Salta, 29 de marzo de 2019

\section{Agradecimientos}

A la comunidad Diaguita- Calchaquí de La Aguada por el apoyo, la compañía y las instancias de intercambio de conocimiento. A los evaluadores anónimos por su aporte, el cual enriqueció este trabajo. A los organizadores del Simposio por invitarnos a participar. Pero sobre todo a Pato, por todas las cosas.

\section{Referencias citadas}

Aljanati, L. I, G. Cochero y S. M. García 2017. “Sedimento de la experiencia histórica?" Etnografías que invitan a la reflexión 
acerca de los patrimonios. Actas de Reunión de Antropología del Mercosur, p 6392.

Arguedas, JM 1951. Notas elementales sobre el arte popular religioso y la cultura mestiza de Huamanga. En: Formación de una cultura nacional indoamericana. Editorial Siglo XXI.148172. México.

Aschero, C. A. 1975. Ensayo para una clasificación morfológica de artefactos líticos. Aplicada a estudios tipológicos comparativos. Informe presentado a CONICET. MS.

Aschero, C. A. 1983. Ensayo para una clasificación morfológica de artefactos líticos, ficha y código descriptivo para artefactos formatizados con rastros complementarios y núcleos. Apéndice B, Cátedra de Ergología y Tecnología, Facultad de Filosofía y Letras, Universidad de Buenos Aires, Buenos Aires.

Barale, A. y D. Delfino. 2007 De los dibujos de la cerámica de una aldea arqueológica a los textiles artesanales: el desarrollo local y la arqueología en Laguna Blanca. Aportes Científicos desde Humanidades 7: 225-235.

Bugallo, L. 2009 Quipildores: Marcas del Rayo en el Espacio de la Puna Jujeña. Cuadernos de la Facultad de Humanidades y Ciencias Sociales 36: 177-202.

Cabral Ortiz, J.E. y M.C. Rivolta 2017. Virke, tinaja o vasija tosca: aproximaciones a un diálogo surgido desde la materialidad Revista de la asociación de arqueólogos profesionales de la república argentina 1 (1): 1-14.

Delfino, D. y P. G. Rodríguez. 1989 Cuando los arqueólogos vienen marchando: interrogantes y propuestas en torno a la defensa y el rescate del patrimonio arqueológico. Revista de Antropología 7: 51-57.

Delfino, D., B. Manasse, R. A. Díaz y M. G. Pisani. 2016. La arqueología socialmente útil y la arqueología pública. Reflexiones desde la praxis. Actas del XIX Congreso Nacional de Arqueología Argentina, Serie monográfica y didáctica 54: 2648-2653. San Miguel de Tucumán.

Delfino, D.; A. Barale; R. A. Díaz; S. J. P. Dupuy; V. E. Espiro; M. G. Pisani. 2013 El Museo Integral de la Reserva de Biosfera de Laguna Blanca como soporte y vehículo de confrontaciones discursivas, de prácticas académicas y campesinas. Actas del IV Encuentro de Museos Universitarios del Mercosur. I Encuentro de Museos Universitarios Latinoamericanos y del Caribe: 150-161. Museo Histórico, Universidad Nacional del Litoral. Santa Fe.

Díaz, P. P. 1972. Sitios Arqueológicos del Valle Calchaquí I. Estudios de Arqueología, Revista del Museo Arqueológico de Cachi 1: pp. 51-59.

Guber, R. 2011 La etnografía: Método, campo y reflexividad. Buenos Aires, Siglo XXI
Hodder, I. 1986 Reading the Past: current approaches to interpretation in Archaeological Method and Theory. Cambridge University press, Cambridge.

Jofré, I.C., S. Biasatti, G. Compañy, G. González, S. Galimberti, N. Najle y P. Aroca. 2008 La cayana: entre lo arqueológico y lo cotidiano. Tensiones y resistencias en las versiones locales del "patrimonio arqueológico" en el norte de San Juan. Relaciones de la Sociedad Argentina de Antropología 23: 181-207.

Juárez G. F. Testimonio kallawaya medicina y ritual en los andes de Bolivia. Hombre y ambiente número monográfico 44-45. Ediciones Abya-Yala. 1997.

Lynch, J., V. Lynch y M. Ríos Malán 2015. Acerca del uso de Pulidores o Litos No Modificados en la producción Cerámica del sitio Villavil (Provincia De Catamarca, Argentina). Arqueología Iberoamericana 27: 33-39.

Manasse, B. 2012 Arqueología en el borde andino del Noroeste Argentino: Sociedades del último milenio en el Valle de Tafí, provincia de Tucumán, República Argentina. Tesis Doctoral. Facultad de Ciencias Naturales y Museo, UNLP. http://naturalis. fcnym.unlp.edu.ar/repositorio/_documentos/tesis/tesis_1212.pdf.

Manasse, B., S. Ibáñez y A. V. Leiva 2019. Arqueología en el este del Mollar (Valle de Tafí, Tucumán). Ampliando historias de los Pueblos Originarios. Libro de Resúmenes del XX Congreso Nacional de Arqueología Argentina. Córdoba, 2019 Universidad Nacional de Córdoba, Argentina: 616- 619.

Menezes, L. 2015. Las cosas están vivas: relaciones entre cultura material, comunidades y legislación arqueológica. Complutum Vol. 26 (1): 37-48.

Mercuri ${ }_{L}$ C., F. I. Coloca y M. N. Weissel 2012. Propuesta de una guía para la clasificación de artefactos líticos históricos. Revista de Arqueología Histórica Argentina y Latinoamericana 6: 45- 61 .

Mc Guire, R. 1987 A Marxist Archaeology. Academic Press, New York.

Pérez, S. 2008. La organización de la tecnología lítica en el Noroeste Argentino. Aproximación a través de experimentación, análisis tecno- morfológico y de microdesgaste por uso de palas y/ o azadas líticas. Comechingonia Virtual 3: 186- 222.

Salerno, M. 2013 Arqueología Pública: reflexiones sobre la construcción de un objeto de estudio. Revista Chilena de Antropología 22: 7-37.

Shanks, M. y C. Tilley 1987 Social theory and archaeology. Polity Press, Oxford.

Vilca, M. 2009. Más allá del "Paisaje". El Espacio de la Puna y Quebrada de Jujuy: ¿Comensal, Anfitrión, Interlocutor? Cuadernos de la Facultad de Humanidades y Ciencias Sociales 36:245-259. 